\title{
Acute erythremic myelosis (true erythroleukaemia): a variant of AML FAB-M6
}

\author{
R P Hasserjian, J Howard, A Wood, K Henry, B Bain
}

\begin{abstract}
Aims-Classic erythroleukaemia (acute myeloid leukaemia M6, or M6 AML) is defined as an excess of myeloblasts in an erythroid predominant background. Leukaemia variants in which the primitive blast cells are demonstrably erythroid are extremely rare and poorly characterised. Variably referred to as "true erythroleukaemia" or "acute erythremic myelosis", they are often included within the M6 AML category even though they do not meet strict criteria for this type of AML. Methods- Two cases of acute erythroid neoplasia are presented with clinical, morphological, immunophenotypic, and cytogenetic analysis.

Results-Both patients presented with profound anaemia, one in a setting of long standing myelodysplasia. Bone marrow examination revealed a predominant population of highly dysplastic erythroid cells in both cases. In one case, the liver was infiltrated by neoplastic erythroid cells. Both patients died within four months of diagnosis.

Conclusions-This report illustrates that cases of acute leukaemia occur in which the dominant neoplastic cell is a primitive erythroid cell without an accompanying increase in myeloblasts. This does not preclude the neoplastic clone originating in a multipotent haemopoietic stem cell, as suggested by cases arising in patients with myelodysplasia. Acute erythremic myelosis should be recognised as a distinct variant of M6 AML.

(f Clin Pathol 2001;54:205-209)
\end{abstract}

Histopathology,

Hospitals NHS Trust,

Du Cane Road,

London W12 0NN, UK

R P Hasseriian

K Henry

Department of

Haematology,

Hammersmith

Hospitals NHS Trust

J Howard

A Wood

Department of

Haematology, St

Mary's Hospital NHS

Trust, Praed St,

London W2 1NY, UK

B Bain

Correspondence to:

Dr Hasserjian

r.hasseriian@ic.ac.uk

Accepted for publication 20 July 2000 The French-American-British (FAB) cation of acute myeloid leukaemia (AML) includes a category, M6 AML, in which there is an excess of myeloblasts in an erythroid dominant marrow. ${ }^{1}$ The erythroblasts are often dysplastic and it is generally accepted that they are part of the neoplastic clone. The FAB classification does not include a category for cases where the neoplastic cells are immature erythroid cells without there being an excess of myeloblasts. However, such cases have been described by others and have been variously designated acute erythromyelosis, acute erythremia, acute erythremic myelosis, erythroblastic leukaemia, erythroleukaemia, Di Guglielmo's syndrome, Di Guglielmo's disease, M6 AML, or M6 AML "variant". ${ }^{2-10}$ We report two additional cases of aggressive acute leukaemia in which the neoplastic cells were clearly erythroid, without an excess of myeloblasts. These two cases are true erythroid neoplasms that should be recognised as a subset of acute leukaemia, as has been proposed recently by the World Health Organisation's preliminary classification of acute leukaemia. ${ }^{11}$

\section{Case reports}

CASE 1

This patient was noted at age 30 in 1977 to have asymptomatic macrocytic anaemia when he was rejected as a volunteer blood donor. At that time, the haemoglobin concentration $(\mathrm{Hb})$ was $122 \mathrm{~g} /$ litre with a mean corpuscular volume (MCV) of $112 \mathrm{fl}$. White blood cell (WBC) and platelet counts were normal. He required no transfusion or other treatment for several years. Past medical history revealed bilateral undescended testicles and hypogonadism, for which he had been receiving testosterone supplements. In 1983, the patient also developed rheumatoid arthritis.

In 1988, dysplastic features (anisocytosis, poikilocytosis, tear drop poikilocytes, and occasional myelocytes and blasts) were noted in the peripheral blood. Bone marrow cytogenetic analysis was normal. In 1992, his anaemia became more severe and bone marrow examination showed trilineage dysplasia consistent with refractory anaemia. By February 1998, his $\mathrm{Hb}$ had fallen to $90 \mathrm{~g} /$ litre and a trial of erythropoietin treatment was begun. His $\mathrm{Hb}$ continued to fall and by August 1998 he had become transfusion dependent. Repeat bone marrow examination at that time showed refractory anaemia with no excess of blasts. However, two weeks later his platelet count, which had previously been normal, had dropped to $29 \times 10^{9} /$ litre. Hepatosplenomegaly developed together with an acute deterioration in liver function. A bone marrow aspirate and trephine biopsy and a transjugular liver needle biopsy were performed.
CASE 2

This 52 year old Somalian man presented to casualty with fatigue and melaena. Over the previous six months he had developed progressive tiredness, bilateral leg swelling, weight loss, and night sweats and, more recently, had noted epistaxis, haemoptysis, and central chest tightness at rest. During the three weeks before presentation he had received weekly blood transfusions, which temporarily relieved the chest tightness. On examination he had widespread bruises and petechiae, mild ankle oedema, and massive splenomegaly. $\mathrm{He}$ was apyrexial, normotensive, and had no 

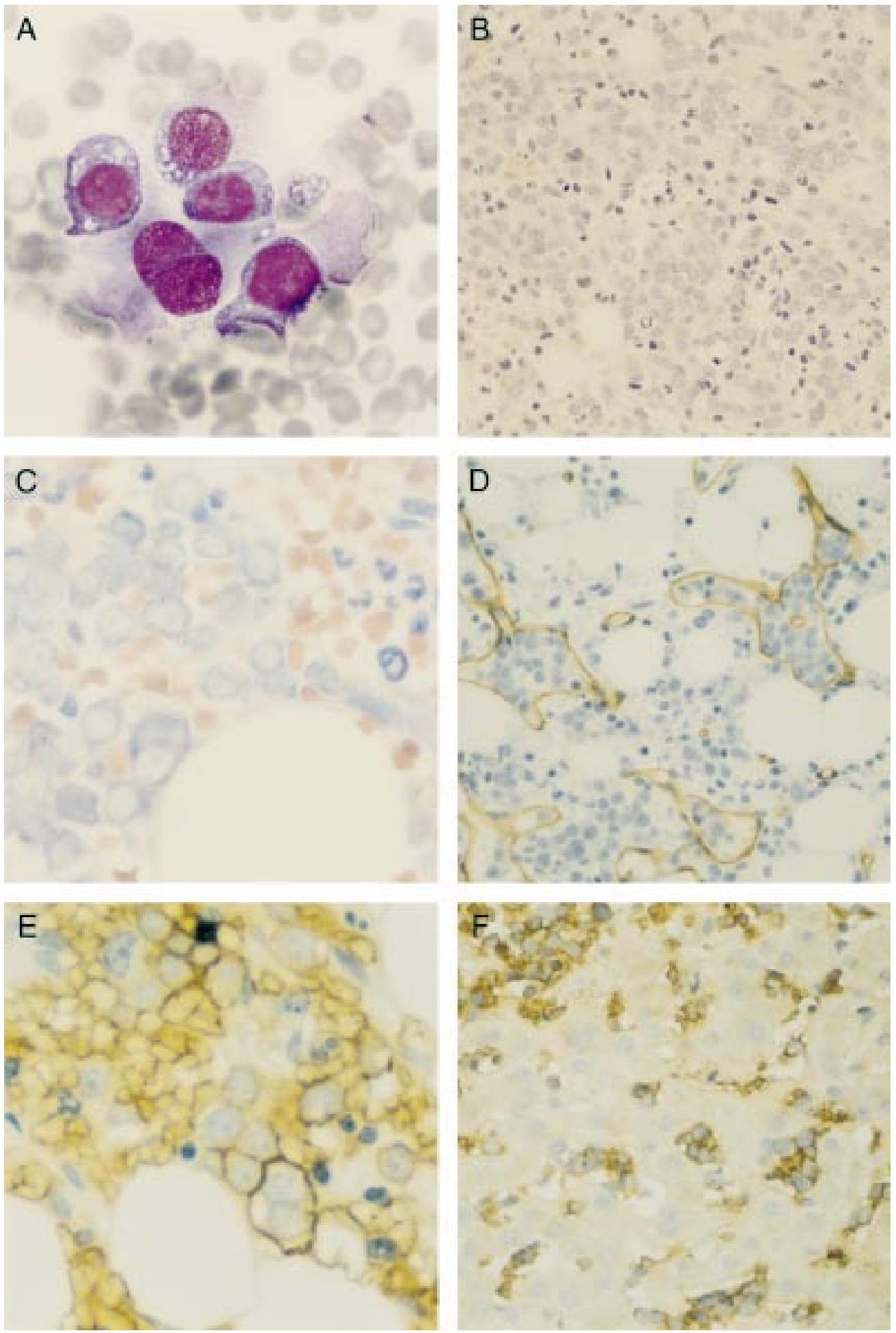

Figure 1 Case 1. (A) Bone marrow aspirate smear showing large primitive cells with vacuolated cytoplasm. (B) The primitive cells comprise about $80 \%$ of the cells of the bone marrow biopsy section, but interspersed maturing myeloid forms are also present (haematoxylin and eosin stained). (C) The primitive cells have basophilic, vacuolated cytoplasm on Giemsa stain; note the maturing myeloid forms in the upper right section. (D) The primitive forms cluster within bone marrow sinuses, as shown by the CD34 immunohistochemical stain highlighting endothelial cells. (E) The immature cells are immunoreactive for $\beta$-sialoglycoprotein; note one binucleate erythroblast. (F) A liver biopsy shows similar cells, immunoreactive for $\beta$-sialoglycoprotein, within hepatic sinusoids.

superficial lymphadenopathy. Full blood count revealed: $\mathrm{Hb}, 80 \mathrm{~g} / \mathrm{litre}$ MCV, 87.3; WBC, $5.0 \times 10^{9} /$ litre, with neutrophil count $2.0 \times 10^{9} /$ litre and platelet count $3 \times 10^{9} /$ litre. Lactate dehydrogenase was greatly raised at $1315 \mathrm{IU} /$ litre and liver function tests were normal. A bone marrow trephine biopsy was performed, but no bone marrow could be aspirated. Peripheral blood was sent for cytogenetic analysis. 

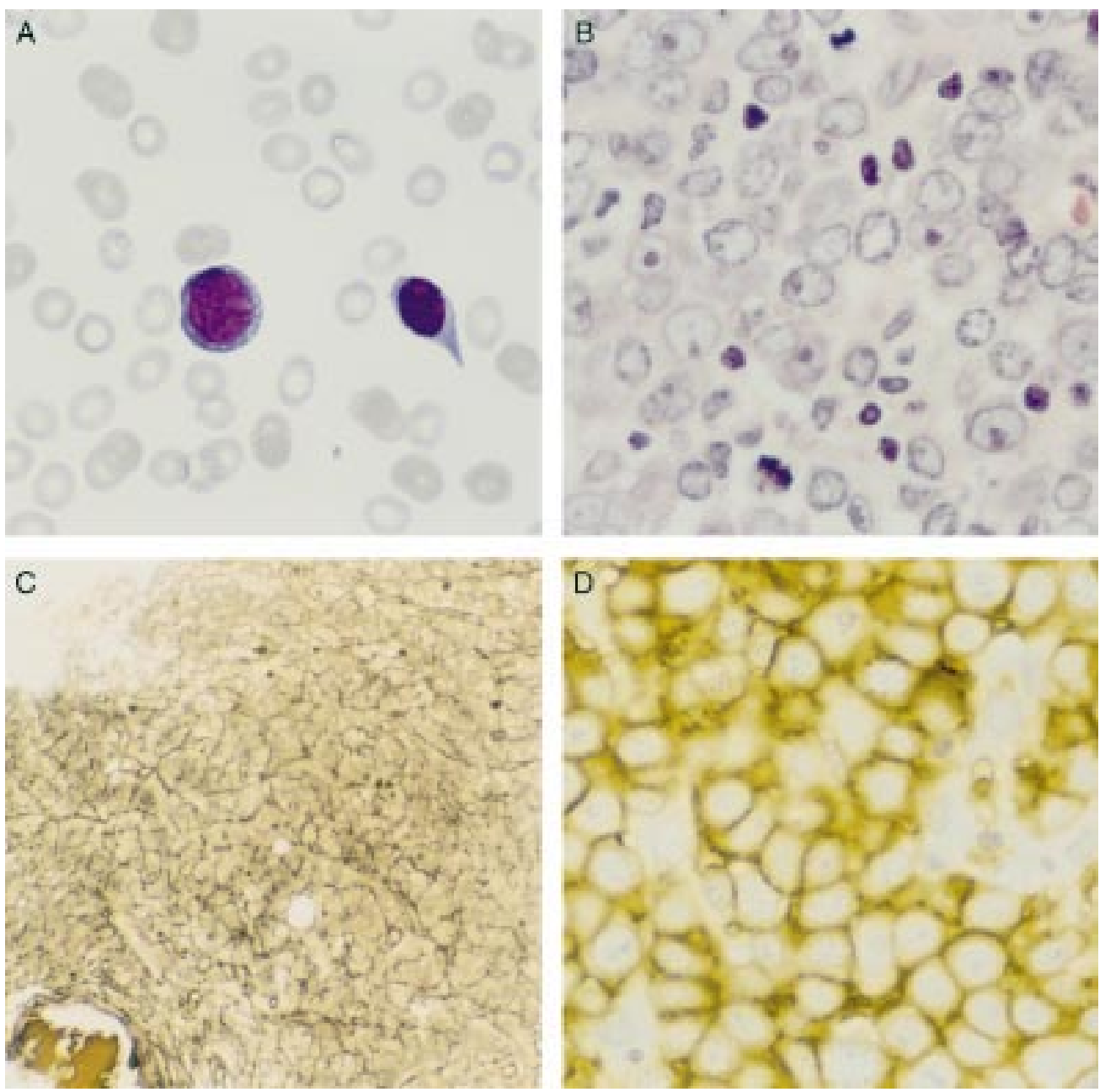

Figure 2 Case 2. (A) Peripheral blood smear showing an immature cell with basophilic vacuolated cytoplasm and a nucleated erythroid cell. (B) Bone marrow biopsy section showing sheets of large cells with vesicular nuclei and prominent nucleoli (haematoxylin and eosin stained). (C) The marrow shows greatly increased reticulin deposition (Gordon and Sweet's silver stain). (D) The immature cells are uniformly immunoreactive for glycophorin $C$.

\section{Methods}

Bone marrow aspirate samples were stained with May-Grünwald-Giemsa. Bone marrow trephine biopsy specimens from the posterior iliac crest were fixed in aceto-zinc-formalin, decalcified in $10 \%$ formic acid, and embedded in paraffin wax. Sections $(3 \mu \mathrm{m})$ were stained with haematoxylin and eosin (H\&E) and Giemsa and silver stain (Gordon and Sweet's method) for reticulin. The liver core biopsy was fixed in $10 \%$ neutral buffered formalin and embedded and stained as above. For immunohistochemistry, dewaxed sections were incubated with the following monoclonal antibodies: CD34 (Qbend; Bionostics, Wyboston, UK), CD61(Clone Y2/51; Dako, Cambridgeshire, UK), CD79a (Dako), neutrophil elastase (Clone NP57; Dako), $\beta$-sialoglycoprotein (Ret40F; a kind gift of David Mason, Oxford, UK), cytokeratin (Cam 5.2; a kind gift of the Imperial Cancer Research Fund, UK), glycophorin C (Dako), CD68 (Clones KP1 and PGM1; Dako), CD138 (BB4; Serotec, Kidlington, UK), CD43 (Serotec), and VS38c (a kind gift of David Mason), or the following polyclonal rabbit antibodies: terminal deoxytransferase (TdT; BioGenix, San Ramon, California, USA), myeloperoxidase (Dako), and
CD3 (Dako). After primary antibody application, slides were developed using a modified streptavidin-biotin technique.

\section{Results}

CASE 1

The bone marrow aspirate was nearly dry, yielding only a few primitive erythroid appearing cells with cytoplasmic vacuolation (fig 1A). The trephine biopsy specimen showed a greatly hypercellular marrow containing sheets of primitive cells comprising over $80 \%$ of the total cells (fig 1B). These cells had round nuclei with finely dispersed chromatin and nucleoli varying from small and round to elongated and large. Cytoplasm was abundant and on Giemsa stain was intensely basophilic (fig 1C). Frequent binucleate and giant forms were seen. One striking feature was the tendency of these primitive cells to form clusters within the bone marrow sinusoids (fig 1D). The remaining cells were an admixture of cells of the granulocytic series, small lymphocytes, and occasional megakaryocytes with hypolobated nuclei. Myeloblasts were not increased in number, and the granulocytic elements showed maturation to neutrophils and only minimal dysplasia. Reticulin was moderately increased (grade 2 of 4 ). 
Immunoperoxidase studies revealed that the primitive marrow cells were immunoreactive for $\beta$-sialoglycoprotein, a highly specific marker of erythroid differentiation ${ }^{12}$ (fig 1E). The cells were CD34, CD20, CD68 (KP1), CD68 (PGM1), myeloperoxidase, neutrophil elastase, and CD61 negative.

The karyotype of bone marrow aspirate on two occasions was $46, \mathrm{XY}$.

The liver core biopsy showed a patchy intrasinusoidal and focal portal infiltrate of large cells, resembling the primitive cells of the trephine biopsy. These cells were $\beta$-sialoglycoprotein positive (fig $1 \mathrm{~F}$ ).

Based on the diagnosis of an erythroleukaemia variant involving bone marrow and liver, the patient was treated with mitoxantrone and oral hydroxyurea, but his transfusion requirement and hepatosplenomegaly increased, and a repeat bone marrow trephine showed persistent erythroid blasts. In early December 1998, he underwent non-myeloablative conditioning (total body irradiation at $200 \mathrm{cGy}$ and cyclosporin $\mathrm{A}$ ) and received an infusion of granulocyte colony stimulating factor (G-CSF) mobilised peripheral blood stem cells from his HLA (human major histocompatibility complex) identical sister. The aim of this treatment was to establish donor-host chimaerism with an anticipated "graft versus leukaemia"effect.

$\mathrm{He}$ developed oliguric renal failure on day 1 post-transplant. He underwent aggressive diuretic and inotropic treatment, but continued to deteriorate. He became hypotensive and unresponsive to treatment and died on day +2 posttransplant. A necropsy was not performed.

CASE 2

The peripheral blood smear showed blasts with mildly basophilic and vacuolated cytoplasm (fig 2A). The bone marrow was variably cellular, with pronounced hypercellular areas alternating with nearly acellular areas (fig $2 B$ ). The former areas comprised sheets of primitive cells with round nuclei, small nucleoli, and moderate amounts of cytoplasm, basophilic on Giemsa stain. Scattered maturing myeloid elements, small lymphocytes, and plasma cells were seen, and megakaryocytes were rare. The reticulin stain showed greatly increased reticulin deposition (grade 3 of 4; fig 2C).
Immunoperoxidase studies showed that the primitive cells were $\beta$-sialoglycoprotein, glycophorin C (fig 2D), and CD43 positive, a small subset (about $5 \%$ ) of these being immunoreactive for CD34. The primitive cells were TdT, CD20, CD68 (KP1), CD68 (PGM1), myeloperoxidase, neutrophil elastase, and CD61 negative.

The karyotype of peripheral blood was 43-44,XY,-5,-7, del(12) (q24),-13,-14, $\operatorname{add}(16)(\mathrm{p} 1),-17,-19, \operatorname{add}(19)(\mathrm{p} 1),+4-5 \mathrm{mar}$ (composite karyotype of fourteen metaphases).

The patient was initially managed with blood and platelet transfusions. Four days after admission he had an episode of symptomatic supraventricular tachycardia which responded to adenosine. Over the next two days he suffered further episodes of chest pain and melaena. Despite continued transfusion support, he died six days after admission from a probable intracranial haemorrhage. A necropsy was not performed.

\section{Discussion}

These two cases represent true erythroid neoplasms in that the neoplastic cells morphologically and immunophenotypically resembled primitive erythroblasts, and myeloblasts were not increased even as a proportion of non-erythroid cells. In fact, although infrequent in both cases, identifiable granulocytic elements showed no or minimal dysplasia. In contrast, the erythroid elements occupied most of the bone marrow and consisted almost exclusively of early and highly dysplastic forms. Cytoplasmic vacuoles (prominent in one of our cases) and erythrophagocytosis by early erythroblasts have been observed in previously reported cases. ${ }^{11}{ }^{13}$ In addition to morphological abnormalities, the early erythroblasts can contain large quantities of haemoglobin $\mathrm{F}^{14}$

Both of our cases had splenomegaly; in case 1, a liver biopsy showed hepatic infiltration by the neoplastic erythroid cells, thus demonstrating the capacity of these cells to behave in a malignant fashion. Di Guglielmo also noted hepatic and splenic infiltration in a patient with pronounced splenomegaly and moderate hepatomegaly. ${ }^{2}$ About one third of previously reported true erythroleukaemia patients (table 1) had clinically recognised hepatosplenomegaly. However, it is interesting to note that

Table 1 Presenting clinical features and survival of current and previously reported true erythroleukaemia cases

\begin{tabular}{|c|c|c|c|c|c|c|}
\hline & No of cases & Splenomegaly & Hepatomegaly & Pancytopenia & $\begin{array}{l}\text { Circulating } \\
\text { proerythroblasts }\end{array}$ & $\begin{array}{l}\text { Survival } \\
\text { (months) }\end{array}$ \\
\hline Di Guglielmo $1928^{2}$ & 1 & $1 / 1$ & $1 / 1$ & $1 / 1$ & $1 / 1$ & 2 \\
\hline Lazzaro $1933^{15}$ & 1 & $1 / 1$ & $1 / 1$ & $0 / 1$ & $1 / 1$ & 0.5 \\
\hline Stodmeister $1941^{16}$ & 1 & $1 / 1$ & $1 / 1$ & $0 / 1$ & $1 / 1$ & 0 \\
\hline Mirchandani et al $1983^{17}$ & 1 & $0 / 1$ & $0 / 1$ & $1 / 1$ & $0 / 1$ & 3 \\
\hline Reiffers et al $1985^{18}$ & 2 & $1 / 2^{\star}$ & $0 / 2^{\star}$ & $2 / 2$ & $1 / 2$ & $0.5,1$ \\
\hline Ruvidic iet al $1986^{5}$ & 1 & ND & ND & $1 / 1$ & $0 / 1$ & 0.5 \\
\hline Griesser and Hornby $1987^{6}$ & 11 & $0 / 11$ & $1 / 11$ & $11 / 11$ & $7 / 11$ & $0.5-4$ \\
\hline Kowal-Vern et al $1992^{19}$ & 13 & ND & ND & ND & ND & $0.1-9$ \\
\hline Tsuji et al $1995^{20}$ & 1 & $1 / 1$ & $1 / 1$ & $1 / 1$ & $0 / 1$ & 2 \\
\hline Mazzella et al $1998^{21}$ & 15 & ND & ND & ND & ND & $0.5-14.5$ \\
\hline Hasserjian et al 2000 & 2 & $2 / 2$ & $1 / 2$ & $2 / 2$ & $1 / 2$ & 0,4 \\
\hline Total & 49 & $7 / 20(35 \%)$ & $6 / 20(30 \%)$ & $19 / 21(90 \%)$ & $12 / 21(57 \%)$ & $1.5^{\star \star}$ \\
\hline
\end{tabular}

*One case had no hepatosplenomegaly at presentation, but did have hepatic, splenic, and renal infiltration with pronormoblasts at necropsy.

$\star \star$ Median of all cases with survival data (40 in total).

$\mathrm{ND}$, not determined or individually reported. 
one case without apparent hepatosplenomegaly did show organ infiltration at necropsy, suggesting that the tendency of neoplastic erythroblasts to involve solid organs might be underestimated.

Cases of conventional erythroleukaemia often occur in the setting of a myelodysplastic syndrome. ${ }^{22}$ Among the reported true erythroleukaemia cases, nearly all patients had pancytopenia. Even in cases with circulating pronormoblasts, these were not usually frequent, the highest reported value being $7 \times 10^{9} / 1$ itre. ${ }^{6}$ In one of the current cases, the erythroleukaemia developed in the setting of a myelodysplastic syndrome. Past medical histories are not provided in most of the previously reported cases, but it is interesting to note that two of the cases in one of the series also had a past history of myelodysplasia. ${ }^{19}$ In addition, chromosome 5 and 7 losses or deletions, often associated with myelodysplastic syndromes, were present in one of our cases, paradoxically in the case without a history of myelodysplasia. Thirteen of 15 previously reported cases with cytogenetic data had losses and/or deletions in the long arms of chromosomes 5 and/or 7 , and several cases had lost material from both of these chromosomes. ${ }^{19}{ }^{21}$ Thus, many true erythroleukaemia cases may share a common pathogenetic mechanism with the more common myelodysplastic syndromes.

The erythroid cells in our cases had an intravascular growth pattern. This intravascular growth and propensity for solid organ infiltration is reminiscent of the rare entity of intravascular large B cell lymphoma, and implies the presence of a vascular adhesion molecule on the surface of these erythroid cells. Examination of more cases of this entity is needed, however, to determine if this is a consistent feature.

Acute leukaemia in which the dominant cell is a primitive erythroid cell, analogous to either a proerythroblast or an early (basophilic) erythroblast, should be recognised as a variant of acute leukaemia. The term "erythroleukaemia" is ambiguous, because it has also been used for M6 AML. Distinction from conventional M6 AML is important, because this true erythroleukaemia has a much poorer prognosis. $^{21}$ The term "M6 variant AML" is suggested. Alternative designations would be acute erythremia or acute erythremic myelosis. In such M6 variant cases, the erythroid component is usually $80-100 \%$ of bone marrow nucleated cells. Although standardised criteria for true erythroleukaemia do not exist, KowalVern et al proposed that cases in which erythroid cells comprise at least $50 \%$ of marrow cells and proerythroblasts comprise greater than $30 \%$ of all erythroid cells should be classified as such..$^{19}$ Our two cases conform to this definition. In contrast to myelodysplastic syndromes with erythroid predominance, the neoplastic cells are primitive and sometimes barely recognisable as erythroid without the aid of immunophenotypic analysis or electron microscopy.

In summary, these cases indicate that true erythroleukaemia is a distinct form of acute leukaemia. Features of these and previously reported cases of this entity suggest an almost universal cytopenic presentation, with no or few circulating erythroblasts and hepatosplenic infiltration. Diagnosis of this unusual form of leukaemia can be difficult because of the lack of an excess of myeloblasts or circulating leukaemic cells. Because these neoplasms appear to behave very aggressively, with a median survival among current and previously reported cases of 1.5 months, prompt diagnosis is essential.

We thank S Parker for excellent technical assistance.

1 Bennett JM , Catovsky D, Daniel MT, et al. Proposed revised criteria for the classification of acute myeloid leukemia. Ann Intern Med 1986;103:620-6.

2 Di Guglielmo G. L'eritremie. Haematologica 1928;9:30147.

3 Schwartz SO, Critchlow J. Erythremic myelosis (di Guglielmo's disease). Critical review with report of four cases, and comments on erythroleukemia. Blood 1952;7:765-98.

4 Kass L. Cytochemical abnormalities of atypical erythroblasts in acute erythremic meylosis. Acta Haematol 1975;54: $321-7$.

5 Ruvidic R, Pavlovic-Kentera V, Boskovic D, et al. Acute erythremic myelosis (Di Guglielmo) following atypical aplastic anemia. Leuk Res 1986;10:451-5.

6 Griesser GH, Horny H-P. Hematopathological features of acute erythremia (morbus Di Guglielmo). A contribution to the classification and differential diagnosis of erythroid neoplasia. Acta Haematol 1987;77:193-7.

7 Brunning RD, McKenna RW. Tumors of the bone marrow. Washington, DC: Armed Forces Institute of Pathology, 1993:75.

8 Bain BJ. Leukaemia diagnosis, 2nd ed. Oxford: Blackwell Science, 1999:25.

9 Mufti GJ, Flandrin G, Schaefer H-E, et al. An atlas of malignant haematology. London: Martin Dunitz, 1996:12.

10 Garand R, Duchayne E, Blanchard D, et al. Minimally differentiated erythroleukaemia (AML M6 “variant"): a rare subset of AML distinct from AML M6. Br F Haematol 1995:90:868-75.

11 Brunning R, Willman CL, Rowley J, et al. Society for Hematopathology: the myelodysplastic syndromes (MDS) and related disorders. Mod Pathol 1999;12:101-6.

12 Gatter KC, Cordell JL, Turley H, et al. The immunohistological detection of platelets, megakaryocytes and thrombi in
routinely processed specimens. Histopathology 1988;13:257.

13 Roggli VL, Saleem A. Erythroleukemia: a study of 15 cases and literature review. Cancer 1982;49:101-8.

14 Forni M, Meyer PR, Levy NB, et al. An immunohistochemical study for hemoglobin A, hemoglobin F, muramidase, and transferrin in erythroid hyperplasia and neoplasia. Am f Clin Pathol 1983;80:145-51.

15 Lazzaro G. Mielosi eritremica acuta. Hematologica 1933;14: 483.

16 Stodmeister R. Akute Erythroblastose und erythroblastische Reaktion. Klin Wehnsehr 1941;20:441.

17 Mirchandani I, Palutke M, Dutcher TF, et al. The value of Mirchandani I, Palutke $M$, Dutcher TF, et al. The value of
immunoperoxidase studies in the diagnosis of 2 cases of acute leukaemia. Scand F Haematol 1983;30:207-10.

18 Reiffers J, Bernard P, Larrue J, et al. Acute erythroblastic leukemia presenting as acute undifferentiated leukemia: a report of two cases with ultrastructural features. Leuk Res 1985;9:413-20.

19 Kowal-Vern A, Cotelingam J, Schumacher HR. The prognostic significance of proerythroblasts in acute erythroleukemia. Am 7 Clin Pathol 1992;98:34-40.

20 Tsuji M, Tamai M, Terada N, et al. Anerythremic form of acute erythremic myelosis (Di Guglilemo's syndrome) causing hepatosplenomegaly due to the infiltration of hemoglobin-bearing blast cells: an autopsy case. Pathol Int 1995;45:310-14.

21 Mazzella FM, Kowal-Vern A, Shrit MA, et al. Acute erythroleukemia: evaluation of 48 cases with reference to classification, cell proliferation, cytogenetics, and prognosis. Am f Clin Pathol 1998;110:590-8.

22 Michiels JJ. Erythroleukemia and myelodysplastic syndromes: an historical appraisal and a personal view. Leuk Lymphoma 1993;9:27-34. 